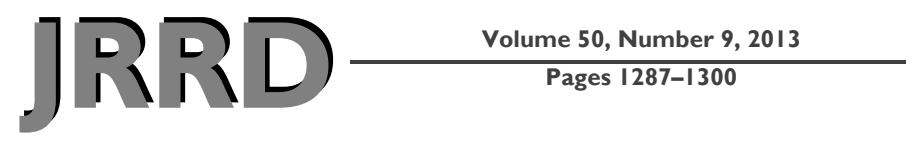

\title{
Power mobility with collision avoidance for older adults: User, caregiver, and prescriber perspectives
}

\author{
Rosalie H. Wang, BSc (OT), PhD; ${ }^{*}$ Alexandra Korotchenko, BHK, MA, PhD(c) ${ }^{2}$ Laura Hurd Clarke, MSW, \\ PhD $;^{2}$ W. Ben Mortenson, BSc (OT), MSc, PhD; ${ }^{3}$ Alex Mihailidis, PhD, PEng ${ }^{1}$ \\ ${ }^{1}$ Intelligent Assistive Technology and Systems Lab, Toronto Rehabilitation Institute-University Health Network and \\ University of Toronto, Toronto, Ontario, Canada; ${ }^{2}$ School of Kinesiology, University of British Columbia, Vancouver, \\ British Columbia, Canada; ${ }^{3}$ Gerontology Research Centre, Simon Fraser University, Vancouver, British Columbia, \\ Canada
}

\begin{abstract}
Collision avoidance technology has the capacity to facilitate safer mobility among older power mobility users with physical, sensory, and cognitive impairments, thus enabling independence for more users. Little is known about consumers' perceptions of collision avoidance. This article draws on interviews (29 users, 5 caregivers, and 10 prescribers) to examine views on design and utilization of this technology. Data analysis identified three themes: "useful situations or contexts," "technology design issues and real-life application," and "appropriateness of collision avoidance technology for a variety of users." Findings support ongoing development of collision avoidance for older adult users. The majority of participants supported the technology and felt that it might benefit current users and users with visual impairments, but might be unsuitable for people with significant cognitive impairments. Some participants voiced concerns regarding the risk for injury with power mobility use and some identified situations where collision avoidance might be beneficial (driving backward, avoiding dynamic obstacles, negotiating outdoor barriers, and learning power mobility use). Design issues include the need for context awareness, reliability, and user interface specifications. User desire to maintain driving autonomy supports development of collaboratively controlled systems. This research lays the groundwork for future development by illustrating consumer requirements for this technology.
\end{abstract}

Key words: caregiver, collision avoidance, intelligent wheelchair, older adult, power mobility, qualitative methods, safety, smart wheelchair, technology design, user experience.

\section{INTRODUCTION}

With increased rates of mobility limitation reported by individuals over age 65 , there has been a dramatic rise in wheeled mobility use in this population [1-2]. Previous research has suggested that power mobility devices (PMDs), which include power wheelchairs and scooters, may benefit older adults by enhancing feelings of competence, autonomy, adaptability, and self-esteem and increasing older users' abilities to engage in a wide range of meaningful physical activities and social roles [3-7].

At the same time, some older adults' access to PMDs may be restricted by their inability to operate PMDs safely and independently [8]. For example, sensory problems (such as visual acuity and visual field deficits) as well as impairments in cognition (including judgment,

\footnotetext{
Abbreviations: $\mathrm{CA}=$ collision avoidance, $\mathrm{CIHR}=$ Canadian Institutes of Health Research, PMD = power mobility device.

*Address all correspondence to Rosalie H. Wang, BSc (OT), PhD; Postdoctoral Fellow, Intelligent Assistive Technology and Systems Lab, Toronto Rehabilitation Institute-University Health Network and Department of Occupational Science and Occupational Therapy, University of Toronto, 160 500 University Ave, Toronto, ON, M5G 1V7 Canada; 416946-8573; fax: 416-946-8570.

Email: rosalie.wang@uhn.ca

http://dx.doi.org/10.1682/JRRD.2012.10.0181
} 
planning, responsiveness to feedback, and decision making) may reduce a driver's ability to respond to hazards [9]. The physical, sensory, and cognitive changes that accompany age-related chronic conditions, such as arthritis, low vision, and dementia, may discourage prescribers from recommending PMDs to older users owing to concerns for injury to self or others [10-11]. Moreover, government funding programs often restrict access to PMDs to those older adults who are able to operate PMDs independently [10,12-13].

Smart or intelligent wheelchairs, which typically combine a conventional power wheelchair with a computer and sensors, have been developed with the aim of enabling people with various disabilities to achieve safe and independent mobility [14]. Smart wheelchairs are designed to assist with obstacle or collision avoidance (CA), navigation, or other functions [14]. Some are designed to operate completely autonomously, without the need for user initiation or intervention, while others use collaborative control, whereby operation is shared between the user and the system [15]. Some of these systems incorporate artificial intelligence that learns about the users' preferences, intentions, and abilities and enables mixed-initiative control [16].

One of the most important intelligent wheelchair functions is obstacle avoidance or CA, which allows a driver to navigate without contacting obstacles in a way that might cause injury or damage property. CA can be designed with varying components and degrees of complexity. Some designs use different primary or multiple sensors, including contact sensors (for example, a bumper skirt [8]) or proximity sensors (for example, ultrasonic or infrared sensors or stereo cameras [14,17-18]). In response to detected obstacles, a control system may (1) deliver a warning to indicate the location and/or proximity of an obstacle, (2) slow the device's movement, or (3) stop movement altogether and require the user to navigate away from the obstacle. The Figure shows an example of an intelligent wheelchair with an obstacleavoidance module.

Attempts to design and evaluate advanced power mobility technology have historically had limited consumer involvement [14,19]. While consumer participation is becoming increasingly supported in medical technology design to improve functionality, usability, safety and, ultimately, acceptance, it continues to be challenging [20-21]. User groups are often heterogeneous, have specific needs, and are problematic to recruit in large numbers. Prototypes are highly specialized and costly to build, and as such, a

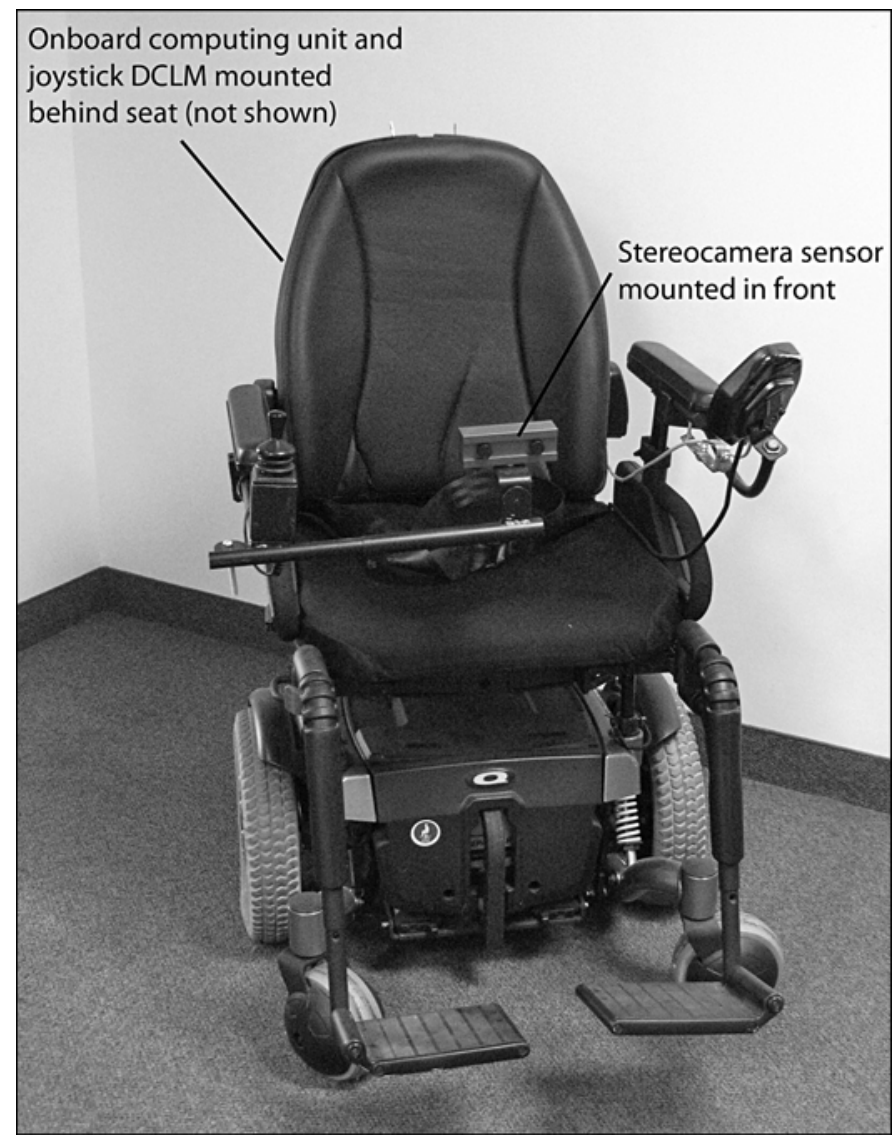

\section{Figure.}

Example of intelligent power wheelchair. Sensor detects features of environment and sends information to onboard computing unit. Onboard computing unit processes information to determine whether to stop or allow wheelchair movement in various directions. If decision is to stop movement in certain directions, joystick direction control logic module (DCLM) will interrupt commands from joystick that direct motor controller to move wheelchair in those directions.

limited number are available for evaluation [22]. Furthermore, developers are frequently disconnected from users' daily driving experiences, creating a mismatch in the goals or priorities of technology developers and the needs and requirements of older PMD users, their caregivers, and PMD prescribers such as occupational therapists or physiotherapists. Therefore, there is a significant gap in knowledge concerning how PMD users, caregivers, and prescribers perceive technology such as CA with respect to its design and utilization. 


\section{METHODS}

In this study, a qualitative research approach was used and thematic analysis was applied to analyze indepth, semistructured interview data. The data reported in this article, which concerns consumers' perceptions of the design and use of CA technology, are a subset of data collected in a larger study focusing on identifying the needs and concerns of older PMD users and the design requirements of intelligent power wheelchair technologies to improve mobility opportunities. The study is part of CanWheel (www.canwheel.ca), an interdisciplinary research program.

\section{Semistructured Interviews}

Interviews were conducted with PMD users, caregivers, and prescribers. The use of semistructured interviews was selected over other data collection approaches for several reasons. Semistructured interviews enabled the collection of rich data on individual experiences and perceptions [23] and allowed the exploration of sensitive topics concerning disability and the body [24]. A comparatively nonthreatening and private environment would likely have been difficult to achieve in group interviews or focus groups. Furthermore, individual interviews were more feasible because of practical issues associated with assembling together older adults with mobility impairments in a group discussion, because the PMD users interviewed were often physically frail, had limited community transportation options, and/or had communication difficulties (for example, had hearing impairment or decreased speech intelligibility or used augmentative communication devices).

Development of the interview schedules for users, caregivers, and prescribers was an iterative and collaborative process involving members of the CanWheel team. The questions pertaining to power mobility technologies were developed with members of the technology development team. The interview schedule for the larger study included questions on the backgrounds of the interviewees, their mobility histories, and experiences with mobility devices. Questions that pertained to power mobility technology in general as well as CA are included in the sample interview guide for users in the Appendix (available online only). The purpose of these questions was to elicit information that might inform the design and use of CA technologies. Because the user and prescriber interviews were completed before the caregiver interviews, there was an opportunity to review the questions for the caregiver group and make modifications to better illustrate the technology concepts. Consequently, caregivers were presented with a short video clip (13 s) showing an individual driving a power wheelchair with CA. The clip illustrated how a power wheelchair might stop and provide an audio prompt to help the user navigate away from a nearby obstacle.

\section{Participant Inclusion and Exclusion Criteria}

All participants had to be able to participate in a spoken or communication device-enabled interview in English. Users were aged 50 or older and had to have used a PMD for at least 12 mo. Caregivers had to be aged 19 or older and be an unpaid caregiver (for example, a family member) or a paid caregiver directly hired by a PMD user aged 50 or older. In addition, caregivers had to provide care for someone who was a current PMD user or who had trialed a PMD. Caregivers of care recipients who had trialed a regular power wheelchair and a power wheelchair with CA in a previous study (up to 10 times of use for both versions of the PMD and over 2-3 wk in total) were also included. Prescribers had to be rehabilitation professionals (occupational therapists or physiotherapists) with recent (within the last $3 \mathrm{yr}$ ) experience prescribing PMDs to people aged 50 or older.

\section{Recruitment}

With use of a nonprobability sampling method, PMD users were recruited via advertisements in community centers, support organization newsletters, clinical contacts, and a community-based contact. Caregivers were recruited via advertisements in a rehabilitation hospital, support organization Web sites, and community centers; a mobility trade show; and a contact conducting power mobility technology research. Prescribers were recruited through third-party recruiters by electronic mail correspondence and by snowball sampling in the prescriber community. User and prescriber interviews were conducted in the Greater Vancouver, Canada, area while caregiver interviews were undertaken in the Greater Toronto, Canada, area.

\section{Procedures}

All study participants provided written informed consent, including permission to digitally record interviews, before interviews commenced. As part of the informed consent process, participants were assured that all data collected from them would remain confidential and 
information revealing their identities would not be released or published. Each of the participants was interviewed at least once at a location of their choosing. Most interviews took place in the participants' place of residence. Others were conducted in their workplace or in a public space such as a coffee shop. Demographic and other descriptive information were collected from participants at the start of the interview. The second author and a trained graduate student conducted the user interviews, the first author conducted the caregiver interviews, and another trained graduate student conducted prescriber interviews. All interviewers were supervised by the study's principal investigator (third author). Participants were offered a \$25 honorarium as compensation for their time.

\section{Data Analysis}

Interviews were transcribed verbatim and analyzed using a thematic analysis approach that aims to identify, reduce, and summarize important patterns and ideas within the data [25]. Transcripts were initially reviewed by three of the authors, one for each data set of users, caregivers, and prescribers, to make preliminary categorizations and to identify data relevant to power mobility safety and CA technology design and use. The relevant interview segments were then compiled and further analyzed by the first author to categorize the data. During this process, data segments were read repeatedly to extract similarities and differences in ideas and experiences that spanned the user, caregiver, and prescriber groups, resulting in the development of a framework of themes and subcategories. To further refine the themes and subcategories, the findings were reviewed through several iterations by the authors. Assumptions and differences in interpretation of the data were discussed until agreement was achieved. The quotes that best illustrated the themes and subcategories in the data were selected for inclusion in the manuscript.

The study used a variety of trustworthiness strategies. Interviewers used postinterview debriefing sessions and reflective commentaries that were shared with the research team to refine interview techniques, discuss initial impressions of the data, identify areas requiring further investigation, and consider alternative approaches and understandings of the data. As a form of investigator triangulation [26], the diverse professional backgrounds of the investigators added to the credibility of the findings. Two authors have backgrounds in occupational therapy, two in the social sciences, and one in engineer- ing. The diversity encouraged a wide variety of interpretations of the data to be considered and questions asked during the data analysis. In order to gain a comprehensive understanding of consumers' perceptions of CA technology and enhance the validity of the findings, data were collected from three data sources (consumer groups). Finally, as a form of member checking, users and prescribers were given an initial report summarizing the findings of the study and were invited to provide feedback; however, none was received.

\section{RESULTS}

\section{Description of Participants}

The age and sex of all participants are shown in Table 1. Additional information about the users $(n=29)$, caregivers $(n=5)$, and prescribers $(n=10)$ are shown in Tables 2, 3, and 4 respectively.

\section{Themes}

Table 5 outlines the three overarching themes and subcategories resulting from our analysis of interviews from the three participant groups.

In the results that follow, participants from different groups are denoted by the following codes: $U=$ users, $\mathrm{C}=$ caregivers, and $\mathrm{P}=$ prescribers, plus their identification numbers. Pseudonyms have been used to protect participant anonymity.

\section{Useful Situations or Contexts}

The majority of users (20 of 29) explicitly indicated that CA might be helpful for them or others. Further, four caregivers and all the prescribers recognized the benefits of CA to assist with safety or functional concerns. Indeed, a large majority of the users (25) and caregivers (4) and all the prescribers (10) readily identified daily situations or contexts in which PMD driving might cause safety or functional problems. Some of the problems included parking and maneuvering in tight spaces such as narrow doors, hallways, and elevators. Areas of specific concern identified by participants and described in more detail below include the following: backing up, avoiding dynamic obstacles, physical barriers to driving outdoors, and learning to use a PMD.

Backing up. Seven users, five prescribers, and one caregiver identified backing up in a PMD as a particular concern and recognized CA as an important potential 
Table 1.

Demographic information of users, caregivers, and prescribers.

\begin{tabular}{|c|c|}
\hline Participant Group & $n$ \\
\hline \multicolumn{2}{|l|}{ Users $(n=29)$} \\
\hline \multicolumn{2}{|l|}{ Age (yr) } \\
\hline 50-59 & 14 \\
\hline $60-69$ & 4 \\
\hline $70-79$ & 3 \\
\hline $80-89$ & 7 \\
\hline $90+$ & 1 \\
\hline \multicolumn{2}{|l|}{ Sex } \\
\hline Male & 14 \\
\hline Female & 15 \\
\hline \multicolumn{2}{|l|}{ Caregivers $(n=5)$} \\
\hline \multicolumn{2}{|l|}{ Age (yr) } \\
\hline 40-49 & 1 \\
\hline $50-59$ & 1 \\
\hline $60-69$ & 2 \\
\hline $70-79$ & 0 \\
\hline $80+$ & 1 \\
\hline \multicolumn{2}{|l|}{ Sex } \\
\hline Male & 0 \\
\hline Female & 5 \\
\hline \multicolumn{2}{|l|}{ Prescribers $(n=10)$} \\
\hline \multicolumn{2}{|l|}{ Age (yr) } \\
\hline 20-29 & 2 \\
\hline 30-39 & 3 \\
\hline $40-49$ & 1 \\
\hline $50-59$ & 2 \\
\hline $60-69$ & 2 \\
\hline \multicolumn{2}{|l|}{ Sex } \\
\hline Male & 1 \\
\hline Female & 9 \\
\hline
\end{tabular}

solution. Paul (U12) and Harold (U23) noted that while mirrors are often recommended, they are inadequate, particularly if they accidentally point to the floor or protrude, making the overall PMD wider and more difficult to maneuver. Users and prescribers agreed that a warning or stopping system would be helpful when backing up to prevent damage to property and harm to people or pets. For example, Nathan (U11) had this to say about being unable to see behind him: "I always worry about running over somebody, 'cause I'm sure this chair would really hurt.” Melissa (P5) and Denise (P10) described how their clients' physical limitations made shoulder checking and backing up particularly challenging, with Denise (P10) remarking that some clients backed up by "sound," that is, stopping only when they heard their PMD colliding with an obstacle. Although Mabel (C5) thought that CA
Table 2.

Descriptive characteristics of users $(n=29)$.

\begin{tabular}{lr}
\hline \multicolumn{1}{c}{ Characteristic } & $\boldsymbol{n}$ \\
\hline Place of Residence & 7 \\
Assisted Living Facility & 12 \\
Long-Term Care Facility & 10 \\
Community Dwelling (private home) & \\
Mobility Device Experience (yr) & 19 \\
1-10 & 4 \\
11-20 & 1 \\
21-30 & 4 \\
31-40 & 1 \\
Undisclosed & \\
Diagnosis Related to Mobility Device Use & 8 \\
Spinal Cord Injury & 4 \\
Multiple Sclerosis & 4 \\
Stroke & 1 \\
Aneurism & 2 \\
Unclassified Neurological Disorder & 5 \\
Poliomyelitis or Postpoliomyelitis & 2 \\
Amputation & 1 \\
Circulation Problem & 1 \\
Urosepsis & 1 \\
Undisclosed & \\
\hline \hline
\end{tabular}

Table 3.

Descriptive characteristics of caregivers $(n=5)$.

\begin{tabular}{ll}
\hline \multicolumn{1}{c}{ Characteristic } & $\boldsymbol{n}$ \\
\hline Relationship to Care Recipient & \\
Spouse (wife) & 2 \\
Privately Hired Caregiver & 1 \\
Lifelong Friend* & 1 \\
Child (daughter) & 1 \\
Years as Caregiver & \\
$0-10$ & 2 \\
$10+$ & 3 \\
\hline *These caregivers provided care to individuals who were not normally power \\
wheelchair users but participated in study to evaluate power wheelchair. \\
\hline
\end{tabular}

would not be particularly useful for her husband at the present time, she agreed that the technology might help her husband back up in his PMD more safely, noting that she often had to alert him to obstacles that were behind him.

Avoiding dynamic obstacles. Twelve users and two prescribers described situations in which dynamic or moving obstacles were problematic. Half the users who identified these types of driving issues did not consider CA to be a useful solution to these problems, while the other half did. For example, Nancy (U6) felt that CA 
Table 4.

Descriptive characteristics of prescribers ( $n=10$, all occupational therapists).

\begin{tabular}{lc}
\hline \multicolumn{1}{c}{ Characteristic } & $\boldsymbol{n}$ \\
\hline Type of Clinical Experience* & \\
Acute & 5 \\
Residential & 6 \\
Rehabilitation & 6 \\
Community & 6 \\
Years of Clinical Experience & \\
1-10 & 4 \\
11-20 & 1 \\
21-30 & 3 \\
31-40 & 2 \\
Years of Power Mobility Experience with & \\
Older Adults & \\
1-10 & 6 \\
11-20 & 1 \\
21-30 & 3 \\
\hline *All prescribers had $>1$ type of clinical experience; therefore, sum of counts $\neq n$.
\end{tabular}

Table 5.

Summary of themes.

\begin{tabular}{cl}
\hline Theme & \multicolumn{1}{c}{ Subcategory } \\
\hline Useful Situations or Contexts & Backing up \\
& Avoiding dynamic obstacles \\
& Physical outdoor barriers \\
& Learning to drive
\end{tabular}

Technology Design Issues and Context awareness

Real-Life Application

System reliability

User interface

Appropriateness of Collision

Avoidance Technology for

Variety of Users

would be helpful for people driving in a busy dining room with numerous people moving among tables and chairs or in a crowded store. Nathan (U11), who also supported CA, mentioned some of the difficulties he typically experienced in anticipating people in motion, recalling several occasions when he nearly hit "people stepping out . . . from hallways going the other direction.” Likewise, Bethany (P4) and Melissa (P5) stated that CA might be useful in busy and unpredictable environments. Bethany (P4) had some reservations about CA, suggesting that "If you're out on a busy sidewalk ... you might be stopping all the time because the pedestrians don't walk around you," but she maintained that CA technology "could help more than hinder.” Indeed, while some users like Lenny (U4) did not specify that CA could help them to navigate dynamic environments, they mentioned actively avoiding crowded situations in which CA might have been useful.

Physical outdoor barriers. Of the users, 19 mentioned environmental obstacles that affected their outdoor driving safety. However, only six of the users indicated that problems with physical outdoor barriers might be avoided or overcome with the help of CA technology. Obstacles included cracked sidewalks, slopes, hidden sidewalk drop-offs, and broken glass. The biggest challenges were negotiating curb drop-offs and curb cuts, with users reporting that they did not notice the drop-off or they took curb cuts at the wrong angle. These incidents also led to tips, occasional falls, and injuries. Jerome (U20) offered a case when a warning system may be useful: "If there was a dip in the road that you couldn't see, if that technology could tell you that the dip is greater than what your chair is able to handle . . . Sometimes when you're talking to someone or you're looking around and you're wheeling ... . all of a sudden the chair dips and it's a huge surprise.”

Learning to drive. The benefits of CA for driver training were identified by two of the users and one prescriber. The value of CA for training purposes was supported by Marcus (U8), who did not think either feature would be useful to him personally, but considered CA to be "a huge training tool" for someone learning about the features and limitations of a new chair. He suggested that older people in particular, who are adapting to new technology, might benefit the most. Noting that adjusting to a PMD's large dimensions could be challenging for some clients, Marlene (P3) echoed this sentiment as she remarked that while CA could be valuable for long-term use, it would be especially beneficial when someone was initially learning to drive.

\section{Technology Design Issues and Real-Life Application}

Regardless of their opinions of CA, users (11), caregivers (2), and prescribers (7) had concerns about the technology's design and how CA might work in practice.

Context awareness. Four users, one caregiver, and three prescribers identified the necessity for context awareness in the system. That is, the CA system would need to know about the driver's intentions and immediate environment and how different obstacles are differentiated. For instance, Bethany (P4) and Gina (C1) wondered 
how a CA system would work in tight spaces. Gina (C1), who was ambivalent about the technology for her husband, noted that the hallways in her home had 3 in. of clearance on either side of her husband's power wheelchair. Because of the limited space, her husband often bumped the walls when turning into their bedroom. Hence, the system would need to know if the user wished to get close to or even needed to make contact with obstacles to achieve mobility in their environment.

In addition to concerns about how close the system would allow users to get to obstacles before a warning was delivered or when the device stopped, some rejected the CA concept based on their understanding of the system requirements for context awareness. Lenny (U4) mentioned situations where he needed to be close to an object, such as a table. Jerome (U20) discussed using object contacts functionally: "With the power wheelchair being so strong and so powerful, if there are obstacles, I can move them by pushing them with my foot pedals . . . and get them out of the way." Further, Valerie (P2) indicated that users might choose to collide with objects in an effort to avoid more serious consequences: "It's just like driving. Sometimes you make a collision to avoid a bigger collision or you hit an object to avoid a person, and you get out of the way of a car coming towards you, even if that means you're hitting something on the sidewalk."

System reliability. Frank (P6) and Paulina (C4) had concerns about a CA system's reliability in detecting obstacles. They wondered how the obstacle detection system would work with respect to sensor placement, how much of the surrounding environment the sensors would cover, and problems with detection failure. Frank (P6), who supported CA for his clients, felt that sensors would need to detect obstacles at a range of heights and all around the chair to provide adequate coverage and ensure reliable detection of obstacles. He gave a personal example of a near collision with a van that had a CA system that failed to detect his car. Paulina (C4) also wondered how much coverage might be necessary to avoid accidents, because sensors would have to detect obstacles at varying heights and curb drop-offs.

User interface. Six users, two caregivers, and five prescribers identified potential user interface issues, which included the degree or nature of the user's shared control with the system, the modalities used to deliver information to the user, and the content and characteristics of the information provided. Both users and prescribers had concerns about the level of control that the user would have over the PMD if it stopped. Users indicated that they wanted to maintain control over their PMDs. Barbara (U18) said that the capability of the PMD to stop by itself would be a great idea, but that "you'd have to be able to override it ... if you looked and saw [the driving situation] was safe." While supportive of the concept, Harold (U23) wondered how long a PMD equipped with CA would be stopped and whether regaining control over the PMD would require intervention from a caregiver or healthcare worker. Jerome (U20) rejected the technology because of the automatic stopping feature, contending "I would perceive that as one more technological thing that someone else or the manufacturer or whoever programs the chair is controlling ... that's how I would see it, as a more of a limitation and restriction and control.”

Questions were also raised regarding the modalities of warnings or prompts given to the user. Participants stressed that the effectiveness of the modality, for example, visual or auditory, would depend on the user's abilities. Lorraine (U1) noted the incompatibility of flashing light indicators with users who were visually impaired. Likewise, Gina (C1) suggested that auditory feedback might not be helpful for individuals with cognitive impairments if they could no longer follow verbal directions, were not spatially oriented, or did not know their right from their left. While Gina (C1) agreed that auditory feedback might be useful for individuals with visual impairment, she was ambivalent about touch feedback because it would have to be delivered on a body location with intact sensation. Her husband had a spinal cord injury and inconsistent sensation below his neck, so tactile feedback could only be applied above his neck.

The content and characteristics of the feedback could limit their usefulness or affect users in various ways. Gina (C1) pointed out that simple audio prompts, such as "turn left," would omit important distance information to avoid an obstacle, information that would be critical for someone with low vision. Janine (P9) suggested that the use of auditory beeps as a warning could be problematic if it did not reveal the reason for the warning going off; for example, if the user does not recognize that there is an obstacle in the environment and the system does not provide obstacle location information to cue the user. Similarly, Marlene (P3) wondered whether, in an extreme case, the "stimulus overload" of a beeper going off frequently and for a reason unknown to the user might agitate the user. Janine (P9) was also concerned whether the information delivery modality might be distracting, 
leading clients to have more accidents. For example, users might need to take their eyes off the road if information was given visually. Nevertheless, Joan (C3) thought that having prompts that indicate the best directions to drive to avoid obstacles might alleviate some of the problem solving and stresses related to driving. According to Denise (P10), the resulting technology would have to be flexible to modify the warning modalities and be programmable to suit different clients.

\section{Appropriateness of Collision Avoidance Technology for Variety of Users}

There were competing views among the users (8), caregivers (4), and prescribers (9) about whether CA would be appropriate for particular users. Melissa (P5) and Frank (P6) thought that all their clients would benefit from having CA. In other cases, users, caregivers, and prescribers offered examples of physical and sensory limitations that could be accommodated with CA technology. Nick (U2), Robena (P8), and Janine (P9) highlighted the value of the technology for people with decreased range of motion in their necks who had difficulty looking behind them to back up. Further, in contrast to those who raised concerns about the applicability of the technology for individuals with visual impairments, five users and two caregivers indicated that the technology would be useful precisely for this population. For example, Mabel (C5) specified that CA could help people who have difficulty judging distances.

Participants were more skeptical of the technology's capacity to accommodate cognitive limitations. Marcus (U8) noted that the technology would be helpful for new drivers and, in particular, those who might have cognitive impairments and resultant difficulties in acquiring new driving skills. At the same time, some prescribers and caregivers viewed cognitive impairment as a complication for power mobility use. Gina (C1), who worked professionally with people with cognitive impairments and who had a family member with dementia, considered power mobility with CA to be inappropriate for individuals with significant cognitive limitations. Similarly, Joan (C3) indicated that the user would have to be able to interpret the system's feedback and follow directions correctly if CA were to be used successfully. Frank (P6) suggested that CA might be beneficial for users who were borderline safe drivers, but not for those with severe cognitive limitations. He explained, "There are those who are clearly unsafe and those that are clearly safe. And in between you have the borderline clients. I wouldn't imagine that you would use CA to make somebody who's in the unsafe category safe. It would be more for those borderline clients, who are already safe, [to] help them be safe longer and decrease chances of accidents.”

\section{DISCUSSION}

In this study, the views of users, caregivers, and prescribers concerning the use of PMDs with CA technology were explored. While the participants expressed realistic concerns about how CA technology might be designed or implemented, overall, they supported the concept of CA. Building on previous reports [27], the findings presented in this study offer justification for the need for CA technology from the perspective of consumers. Discussion of the results examines the driving concerns experienced or observed by participants, CA technology design issues, the potential users of CA technology, recommendations for future work, and the limitations of the study.

\section{Power Mobility Driving Concerns}

Participants identified a variety of potential safety concerns with power mobility use, but also emphasized how critical and challenging it was to carry out daily activities in desired locations with PMDs. Regarding safety, the risk of potentially injuring others was a key concern for participants and an issue that CA was perceived to have the potential to ameliorate. PMD users' and prescribers' concerns regarding driving into and injuring others with PMDs are similarly highlighted in other studies [7,28-29]. Likewise, the physical environmental barriers to safe and functional mobility experienced in everyday life described by participants in this study are comparable to those identified in others studies [29-31]. These findings suggest that while PMDs may enable mobility and enhance users' lives in a number of ways $[4,13,30-31]$, bodily limitations, the physical and social environment, and suboptimal technology continue to leave many PMD users' needs and concerns unaddressed. Indeed, current technology may inadequately address users' requirements because it is also unclear whether many of the power wheelchairs currently available on the market are meeting the needs of older users, given that the average age of power wheelchair users is approximately 51 yr [32]. Furthermore, some driving safety and functional mobility concerns may be 
addressed with improved provision practices. For example, some users may have insufficient guidance or supervision from healthcare providers in their acquisition of a PMD. That is, users may purchase devices without appropriate assessment or training [31,33-34]. Suffice it to say, many opportunities exist for advanced technology and better service delivery to improve the lives of older PMD users.

Although most of the users who identified daily driving concerns also acknowledged the potential of advanced technology to address these issues, many did not. There may be several possible explanations for this discrepancy. For example, the participants' ambivalence toward the technology may have been related to how CA was explained to participants, resulting in different understandings of how the technology may operate. Users may have had pre-existing beliefs or assumptions about how the technology operated. Their attitudes toward assistive technology and openness to or awareness of technological possibilities may have also been a factor [35-36]. Moreover, users may have been wary of highlighting driving difficulties for fear of putting their driving privileges at risk.

While CA was presented as a PMD modification for long-term use, participants also identified the utility of the technology during the learning period of PMD use. Previous studies have suggested that new PMD users with and without intact sensory, perceptual, or cognitive abilities may benefit from additional training in device use [37-38]. In another study, some older users noted that they did not use their PMDs as frequently as they would like because of their of lack confidence in their abilities to negotiate the environment [29]. Hence, CA technology may provide enhanced opportunities for skills and confidence training. CA technology's potential as an adjunctive training tool for older users has been previously suggested [39]. However, the effectiveness of CA and the recommended protocols to be used in power mobility training with older adults requires further exploration.

\section{Technology Design Issues}

Despite the support the majority of the participants displayed for the concept of CA, many raised issues that reflected the persistent challenges in the technical implementation of CA technology that have been noted in the literature. While possibly unknown to the participants, these technical concerns are being actively researched in the fields of robotics and artificial intelligence for power mobility and other applications [40-41]. Indeed, in power mobility applications, the requirements are stringent since a failure can result in serious injury to bystanders or users [8]. Achieving sufficient sensor sensitivity and reliability for different obstacle types, materials, and locations and enabling robust detection in natural and varied lighting are important challenges to be addressed [42]. The participants identified several driving concerns, such as dynamic obstacle avoidance and negotiating physical outdoor obstacles that are ongoing issues [4344]. Furthermore, researchers are developing contextaware systems and participants recognized the importance of context awareness, including the need for a decision-making hierarchy that recognizes and prioritizes avoidance of people as opposed to inanimate objects. When trying to create a truly functional CA system, a key consideration voiced by a number of participants was the need for a collaboratively controlled system that allows the user to maintain as much control over the operation of the PMD as possible [15,45-46]. Much more research is necessary to elucidate the detailed requirements of how to match technology to specific users with collaborative control and other user interface strategies. However, because of the complexity of these functions, the development and integration of these capabilities into a complete power mobility CA system for users has yet to be achieved.

\section{Potential Users of Collision Avoidance Technology}

Findings suggest that CA technology should not be developed exclusively for users with physical, sensory, and/or cognitive challenges that currently preclude power mobility use $[8,41]$. Rather, CA technology can be applicable to a broad range of user abilities and requirements, including current PMD users who might benefit from the technology as an added safety feature. As identified by one of the prescribers in this study, it is conceivable that CA may assist users whose needs and abilities change over time, thereby prolonging their use of PMDs. With the potential for enhanced safety, the use of power mobility could also be extended to a larger number of users. As previously suggested in the literature, however, it is difficult to determine the need for such technology (for example, the number of users who might benefit) because capable PMD users and nondisabled people may occasionally have accidents [7,27].

The criteria for PMD prescription and use often require intact cognition, and cognitive limitation has been 
found in one study to be the determining factor in whether an older adult is prescribed a manual over a power wheelchair [47]. However, the user, caregiver, and prescriber groups' accounts of their experiences of power mobility suggest that users' capacities may lie along a continuum of cognitive abilities. That is, the accounts of the participants in this study indicate that there may be existing cases of users with mild or even moderate cognitive impairment operating PMDs. The prescribers alluded to situations wherein some clients continued to utilize power mobility even though they were considered to be borderline safe drivers. These observations correspond with those found in the study by Mortenson et al. [7], which reported that stakeholders from a variety of groups (residents, families, and staff) were in favor of giving the opportunity to use power mobility in residential care even following the emergence of symptoms of cognitive impairment.

In contrast, in discussing technological modifications that may enable safe and independent use, participants highlighted their reservations regarding the potential for CA to enable power mobility use among individuals with cognitive impairments. To begin, some participants voiced concerns over whether the technology can adequately compensate for cognitive changes and minimize the risk for serious injury to others and the driver. For instance, it could be argued that users with cognitive impairments might inadvertently place themselves in high-risk situations in which CA technology is unable to negotiate the environment. The concern also leads to questions of informed consent and liability and the extent to which a user might knowingly consent to using a PMD that might cause injury or harm. Further, participants raised questions regarding the severity of cognitive decline that might be accommodated with CA technology. It remains unclear what features, modifications, and user interface requirements are necessary to enable users with cognitive impairments to use a power wheelchair safely and effectively. The case for power mobility for users with cognitive impairment remains complicated and further exploration is necessary to better define how and under what circumstances the technology might be used.

\section{Recommendations for Future Work}

The general support for the idea of CA technology provides encouragement for the continued collaboration between clinicians, engineers, and computer scientists and users, caregivers, and prescribers so as to overcome the outstanding technical challenges and to address the needs and requirements of consumers presented in this article. Much of the development work in CA technology has not been consumer driven or has not involved the consumer groups who were involved in this study. These findings may begin to fill knowledge gaps in the development process for CA technology for older adult PMD users.

In spite of the difficulties inherent in consumer collaboration, involving various consumer groups in CA development offers a number of benefits and should be pursued in future work. The difficulties often cited in involving consumers in technology design include time and funding constraints, complications in recruiting participants, consumers' lack of understanding of how complex technologies work, consumers' vulnerability and intolerance for demanding activities, and developers' negative perceptions of the usefulness or validity of consumer views [21]. Some users may experience communication difficulties as a result of physical or cognitive disabilities, which may further restrict effective collaboration $[20,48]$. However, the benefits of involving consumers were apparent in this study. Diverse participant groups offered valuable insights into the applicability of CA technology to their lives and made astute observations and queries critical to informing the design of future power mobility technology. The insights gained can help to improve the relevance, usefulness, and adoption of CA. The constructive comments offered by the participants also help to dispel concerns that users of assistive technology may not have much to contribute to the design process [21].

With respect to the technology design process and methodology to evaluate future prototypes, it is recommended that future studies involve users, caregivers, and prescribers in more hands-on experiences in trialing prototype systems. Verbal explanations and video demonstrations may offer good stimuli for discussion, as used in the current study, but real tests of usefulness, usability, and environmental fit can be best performed when consumers try working prototypes and experience the functions and implications of using CA themselves. Furthermore, because the development process involves multiple iterations of hardware and software implementations, it will be crucial to involve user groups at multiple stages, rather than when the development team considers a product to be "finished" [21,49]. Testing at these end points may be detrimental to resources and morale if the focus of development diverges too greatly from what 
consumers groups may need or want and discovered only at the time of evaluation. Major design changes may also be less likely to occur if identified at later stages [49].

Finally, several other avenues for future research are highlighted in this article. Additional research directions relate to the continual need to pursue new technology and service delivery practices to address PMD use safety and functional mobility issues for current and prospective older adult users. Specifically, further exploration of effective training procedures for PMD use and the application of CA technology in training are warranted. As CA and other technology are being developed to potentially expand the user base for PMDs, it will be necessary to investigate the implications of users' cognitive abilities and the associated technology design and service provision requirements.

\section{Study Limitations}

There were several limitations to this study. The participants were volunteers who were made aware of the study by advertisements and clinical or other contacts. While efforts were made to distribute the information broadly, there was a potential for volunteer bias, leading to limitations in the applicability of the findings to some consumer groups. However, the broad participant inclusion criteria within each of the groups may improve the transferability of findings [50]. For example, users who lived in various types of residences and who had a range of diagnoses that affect mobility were included. Caregivers included those who cared for someone living at home or in a care facility. Prescribers included those who worked in a range of practice areas. The team initially planned to simultaneously recruit caregivers associated with users, but this proved challenging. Despite expanding the recruitment to include caregivers who were not associated with a user already involved in the study, the team found it challenging to recruit caregivers at the site of the user and prescriber interviews. This lead to the involvement of a second study location where, despite further efforts, it was only possible to recruit five caregivers. Because the caregiver interviews were conducted following the completion of the user and prescriber interviews, it was determined to be useful during the caregiver interviews to include a video illustrating how the technology might work. As such, the explanation of the technology may have been clearer for the caregivers. Furthermore, the two caregivers of users who were not current PMD users may have had different perspectives and knowledge about power mobil- ity. For example, they may not have had the knowledge of long-term use that other caregivers may have had. However, caregivers in these cases were included because it was felt that they had sufficient knowledge about power mobility and their care recipients' needs to be included in the study. Because the caregivers had some experience with CA technology, their perspectives of the technology may also have differed. It may also have been challenging for participants to evaluate a new technology without a functional prototype being present. Responses were thus based on hypothetical technology and dependent on how it was presented and interpreted. Finally, this interviewbased study explored design requirements as themes and subcategories, and as such, participants were not asked to prioritize their technology-related needs and requirements. Nevertheless, the findings are valuable in formulating use cases or scenarios to guide design [51] and to offer support for existing research and development efforts.

\section{CONCLUSIONS}

The findings from this study indicate that the development of CA for older adults should continue to be pursued. The majority of participants interviewed were supportive of CA and thought that the technology might be beneficial to current PMD users and users with visual impairments, but might be unsuitable for people with significant cognitive impairments. Important technology design issues included the need for context awareness in the intelligent system, reliability, and user interface specifications. Furthermore, the desire for users to maintain as much autonomy over their driving as possible supports the need for a collaboratively controlled system. This research lays the groundwork for future development by identifying and illustrating consumer needs for this technology.

\section{ACKNOWLEDGMENTS}

\section{Author Contributions:}

Study conception and design: A. Korotchenko, L. Hurd Clarke.

Data acquisition: R. H. Wang, A. Korotchenko.

Analysis and interpretation of data: R. H. Wang, A. Korotchenko, L. Hurd Clarke, W. B. Mortenson, A. Mihailidis.

Manuscript preparation and revisions: R. H. Wang, A. Korotchenko, L. Hurd Clarke, W. B. Mortenson, A. Mihailidis.

Financial Disclosures: The authors have declared that no competing interests exist. 
Funding/Support: This material was based on work supported by a Canadian Institutes of Health Research (CIHR) CanWheel Emerging Team grant in Wheeled Mobility for Older Adults (grant AMG100925). Funding for Dr. Wang is provided by a CIHR Postdoctoral Fellowship Award. Ms. Korotchenko's work is supported by a Social Sciences and Humanities Research Council of Canada, Canada Graduate Scholarship Doctoral Award. Dr. Mortenson's work was supported by a CIHR Banting Postdoctoral Fellowship Award.

Additional Contributions: The authors thank the PMD users, caregivers, and prescribers who were interviewed. Additionally, the authors acknowledge the help of Erica Bennett, Krista Best, and Pooja Viswanathan in conducting interviews and of other members of the CanWheel team for their assistance with developing the interview schedules. W. Ben Mortenson is now an assistant professor at the University of British Columbia.

Institutional Review: Ethical approval for the study was granted by the University of British Columbia Behavioural Research Ethics Board and Toronto Rehabilitation Institute Research Ethics Board. Written informed consent was provided by all study participants. Participant Follow-Up: The authors do not plan to inform participants of the publication of this study.

\section{REFERENCES}

1. Shumway-Cook A, Ciol MA, Yorkston KM, Hoffman JM, Chan L. Mobility limitations in the Medicare population: Prevalence and sociodemographic and clinical correlates. J Am Geriatr Soc. 2005;53(7):1217-21. [PMID:16108942] http://dx.doi.org/10.1111/j.1532-5415.2005.53372.x

2. Statistics Canada. Participation and activity limitation survey 2006: Analytical report (Report No. 89-628-XIE). Ottawa (Canada): Government of Canada, Minister of Industry; 2007.

3. Auger C, Demers L, Gélinas I, Miller WC, Jutai JW, Noreau L. Life-space mobility of middle-aged and older adults at various stages of usage of power mobility devices. Arch Phys Med Rehabil. 2010;91(5):765-73. [PMID:20434615]

http://dx.doi.org/10.1016/j.apmr.2010.01.018

4. Brandt Å, Iwarsson S, Ståhle A. Older people's use of powered wheelchairs for activity and participation. J Rehabil Med. 2004;36(2):70-77. [PMID:15180221] http://dx.doi.org/10.1080/16501970310017432

5. Buning ME, Angelo JA, Schmeler MR. Occupational performance and the transition to powered mobility: A pilot study. Am J Occup Ther. 2001;55(3):339-44. [PMID:11723976] http://dx.doi.org/10.5014/ajot.55.3.339

6. Clarke P, Colantonio A. Wheelchair use among community-dwelling older adults: Prevalence and risk factors in a national sample. Can J Aging. 2005;24(2):191-98.

[PMID:16082621]

http://dx.doi.org/10.1353/cja.2005.0059
7. Mortenson WB, Miller WC, Boily J, Steele B, Odell L, Crawford EM, Desharnais G. Perceptions of power mobility use and safety within residential facilities. Can J Occup Ther. 2005;72(3):142-52. [PMID:15988961] http://dx.doi.org/10.1177/000841740507200302

8. Wang RH, Gorski SM, Holliday PJ, Fernie GR. Evaluation of a contact sensor skirt for an anti-collision power wheelchair for older adult nursing home residents with dementia: Safety and mobility. Assist Technol. 2011;23(3):117-34. http://dx.doi.org/10.1080/10400435.2010.541406

9. Hardy P. Powered wheelchair mobility: An occupational performance evaluation perspective. Aust Occup Ther J. 2004;51:34-42.

http://dx.doi.org/10.1111/j.1440-1630.2004.00413.x

10. Hardy P. Examining the barriers: Powered wheelchair mobility for people with cognitive and/or sensory impairments. Australian Rehabilitation and Assistive Technology Association National Conference; 2004 Jun 2-4; Melbourne, Australia.

11. Mortenson WB, Clarke LH, Best K. Prescribers' experiences with powered mobility prescription among older adults. Am J Occup Ther. 2013;67(1):100-107.

[PMID:23245788]

http://dx.doi.org/10.5014/ajot.2013.006122

12. Ontario Assistive Devices Program (ADP). Wheelchair, positioning and ambulation aids, policy and administration manual [Internet]. Ontario (Canada): Ontario Ministry of Health and Long-Term Care; 2012 Mar: Available from: http://www.health.gov.on.ca/en/pro/programs/adp/ information_technology/docs/ mobility policy administration manual.pdf

13. Frank AO, Ward J, Orwell NJ, McCullagh C, Belcher M. Introduction of a new NHS electric-powered indoor/outdoor chair (EPIOC) service: benefits, risks and implications for prescribers. Clin Rehabil. 2000;14(6):665-73. [PMID:11128743] http://dx.doi.org/10.1191/0269215500cr376oa

14. Simpson RC. Smart wheelchairs: A literature review. J Rehabil Res Dev. 2005;42(4):423-36. [PMID:16320139] http://dx.doi.org/10.1682/JRRD.2004.08.0101

15. Urdiales C, Fernández-Carmona M, Peula JM, Cortés U, Annichiaricco R, Caltagirone C, Sandoval F. Wheelchair collaborative control for disabled users navigating indoors. Artif Intell Med. 2011;52(3):177-91. [PMID:21723104] http://dx.doi.org/10.1016/j.artmed.2011.05.002

16. Mihailidis A, Elinas P, Boger J, Hoey J. An intelligent powered wheelchair to enable mobility of cognitively impaired older adults: An anticollision system. IEEE Trans Neural Syst Rehabil Eng. 2007;15(1):136-43. [PMID:17436886] http://dx.doi.org/10.1109/TNSRE.2007.891385 
17. LoPresti E, Simpson R, Miller D, Nourbakhsh I. Evaluation of sensors for a smart wheelchair. Rehabilitation Engineering \& Assistive Technology Society Annual Conference; 2002 Jun; Orlando, FL.

18. Viswanathan P, Boger J, Hoey J, Mihailidis A. A comparison of stereovision and infrared as sensors for an anti-collision powered wheelchair for older adults with cognitive impairments. 2nd International Conference on Technology and Aging; 2007 Jun; Toronto, Canada.

19. Wang RH. Enabling power wheelchair mobility with longterm care home residents with cognitive impairments. Toronto (Canada): University of Toronto; 2011.

20. Martin JL, Murphy E, Crowe JA, Norris BJ. Capturing user requirements in medical device development: The role of ergonomics. Physiol Meas. 2006;27(8):R49-62. [PMID:16772664] http://dx.doi.org/10.1088/0967-3334/27/8/R01

21. Shah SG, Robinson I. Benefits of and barriers to involving users in medical device technology development and evaluation. Int J Technol Assess Health Care. 2007;23(1): 13137. [PMID:17234027]

http://dx.doi.org/10.1017/S0266462307051677

22. Tsui K, Yanco H. Towards establishing clinical credibility for rehabilitation and assistive robots through experimental design. Robotics: Science and Systems Workshop on Good Experimental Methodology in Robotics; 2009 Jun 28; Seattle, WA.

23. Holstein MB, Gubrium JF. Handbook of interview research: Context and method. Thousand Oaks (CA): SAGE Publications; 2002.

24. Clarke LH. Overcoming ambivalence: The challenges of exploring socially charged issues. Qual Health Res. 2003; 13(5):718-35. [PMID:12756690] http://dx.doi.org/10.1177/1049732303013005009

25. Ayres L. Thematic coding and analysis. In: Given L, editor. The SAGE encyclopedia of qualitative research methods. Thousand Oaks (CA): SAGE Publications; 2008. p. 868-69.

26. Rothbauer PM. Triangulation. In: Given L, editor. The SAGE encyclopedia of qualitative research methods. Thousand Oaks (CA): SAGE Publications; 2008. p. 893-95.

27. Simpson RC, LoPresti EF, Cooper RA. How many people would benefit from a smart wheelchair? J Rehabil Res Dev. 2008;45(1):53-71. [PMID:18566926]

http://dx.doi.org/10.1682/JRRD.2007.01.0015

28. Mendoza RJ, Pittenger DJ, Saftler Savage F, Weinstein CS. A protocol for assessment of risk in wheelchair driving within a healthcare facility. Disabil Rehabil. 2003;25(10): 520-26. [PMID:12745963] http://dx.doi.org/10.1080/0963828031000090515

29. Evans S, Frank AO, Neophytou C, de Souza LH. Older adults' use of, and satisfaction with, electric powered indoor/ outdoor wheelchairs. Age Ageing. 2007;36(4):431-35.

\section{[PMID:17384418]}

http://dx.doi.org/10.1093/ageing/afm034

30. May M, Rugg S. Electrically powered indoor/outdoor wheelchairs: Recipients' views of their effects on occupational performance and quality of life. Br J Occup Ther. 2010;73(1):2-12.

http://dx.doi.org/10.4276/030802210X12629548272583

31. Edwards K, McCluskey A. A survey of adult power wheelchair and scooter users. Disabil Rehabil Assist Technol. 2010;5(6):411-19. [PMID:20450455]

http://dx.doi.org/10.3109/17483101003793412

32. Fitzgerald SG, Kelleher A, Teodorski E, Collins DM, Boninger $\mathrm{M}$, Cooper RA. The development of a nationwide registry of wheelchair users. Disabil Rehabil Assist Technol. 2007;2(6):358-65. [PMID:19263566]

http://dx.doi.org/10.1080/17483100701745752

33. Frank A, Neophytou C, Frank J, de Souza L. Electric-powered indoor/outdoor wheelchairs (EPIOCs): users' views of influence on family, friends and carers. Disabil Rehabil Assist Technol. 2010;5(5):327-38. [PMID:20377422] http://dx.doi.org/10.3109/17483101003746352

34. Greer N, Brasure M, Wilt TJ. Wheeled mobility (wheelchair) service delivery: Scope of the evidence. Ann Intern Med. 2012;156(2):141-46. [PMID:22250145] http://dx.doi.org/10.7326/0003-4819-156-2-201201170$\underline{00010}$

35. Robertson S. Requirements trawling: Techniques for discovering requirements. Int J Hum Comput Stud. 2001; 55(4):405-21. http://dx.doi.org/10.1006/ijhc.2001.0481

36. Heinz M, Martin P, Margrett JA, Yearns M, Franke W, Yang HI, Wong J, Chang CK. Perceptions of technology among older adults. J Gerontol Nurs. 2013;39(1):42-51. [PMID:23244061]

http://dx.doi.org/10.3928/00989134-20121204-04

37. Nitz JC. Evidence from a cohort of able bodied adults to support the need for driver training for motorized scooters before community participation. Patient Educ Couns. 2008; 70(2):276-80. [PMID:18065187]

http://dx.doi.org/10.1016/j.pec.2007.10.014

38. Mountain AD, Kirby RL, Eskes GA, Smith C, Duncan H, MacLeod DA, Thompson K. Ability of people with stroke to learn powered wheelchair skills: A pilot study. Arch Phys Med Rehabil. 2010;91(4):596-601.

[PMID:20382293]

http://dx.doi.org/10.1016/j.apmr.2009.12.011

39. Wang RH, Mihailidis A, Dutta T, Fernie GR. Usability testing of multimodal feedback interface and simulated collision-avoidance power wheelchair for long-term-care home residents with cognitive impairments. J Rehabil Res Dev. 2011;48(7):801-22. [PMID:21938666] http://dx.doi.org/10.1682/JRRD.2010.08.0147 
40. Kumar V, Bekey G, Zheng Y. Industrial, personal, and service robots. In: Bekey G, Ambrose R, Kumar V, Lavery D, Sanderson A, Wilcox B, Yuh J, Zheng Y, editors. Robotics: State of the art and future challenges. London (UK): Imperial College Press; 2008. p. 89-101.

41. Lopresti EF, Sharma V, Simpson RC, Mostowy LC. Performance testing of collision-avoidance system for power wheelchairs. J Rehabil Res Dev. 2011;48(5):529-44. [PMID:21674403] http://dx.doi.org/10.1682/JRRD.2010.01.0008

42. Sharma VK. Design and evaluation of a distributed, shared control, navigation assistance system for power wheelchairs. Pittsburgh (PA): University of Pittsburgh; 2009.

43. Suzuki T, Hashimoto M, Takahashi K. Laser-based road recognition for a smart electric wheelchair. IEEE International Conference on Robotics and Biomimetics; 2011 Dec 7-11; Phuket Island, Thailand.

44. Coughlan J, Manduchi R, Shen H. Computer vision-based terrain sensors for blind wheelchair users. 10th International Conference on Computers Helping People with Special Needs; 2006 Jul 11-13; Linz, Austria.

45. Carlson T, Demiris Y. Collaborative control for a robotic wheelchair: Evaluation of performance, attention, and workload. IEEE Trans Syst Man Cybern B Cybern. 2012; 42(3):876-88. [PMID:22275718] http://dx.doi.org/10.1109/TSMCB.2011.2181833

46. Parikh S, Grassi VJ, Kumar VJ, Okamoto JJ. Integrating human inputs with autonomous behaviours on an intelligent wheelchair platform. IEEE Trans Intell Transp Syst. 2007;22(2):33-41. http://dx.doi.org/10.1109/MIS.2007.36

47. Karmarkar AM, Dicianno BE, Graham JE, Cooper R, Kelleher A, Cooper RA. Factors associated with provision of wheelchairs in older adults. Assist Technol. 2012;24(3): 155-67. [PMID:23033733] http://dx.doi.org/10.1080/10400435.2012.659795

48. Magnier C, Thomann G, Villeneuve F, Zwolinski P. Methods for designing assistive devices extracted from 16 case studies in the literature. Int J Interac Des Manuf. 2012; 6(2):93-100. http://dx.doi.org/10.1007/s12008-012-0143-2

49. Parent AS, Georgantzi N, Gheno I. Ensuring a fruitful future to innovation and research: Practical guidance for the involvement of older people in research. 10th International Conference on Smart Homes and Health Telematics; 2012 Jun 12-15; Artimino, Italy.

50. Jensen D. Transferability. In: Given L, editor. The SAGE encyclopedia of qualitative research methods. Thousand Oaks (CA): SAGE Publications; 2008. p. 887.

51. Rosson MB, Carroll JM. Scenario-based design. In: Jacko J, Sears A, editors. The human-computer interaction handbook: Fundamentals, evolving technologies and emerging applications. Mahwah (NJ): Lawrence Erlbaum Associates; 2002. p. 1032-50.

Submitted for publication October 9, 2012. Accepted in revised form March 19, 2013.

This article and any supplementary material should be cited as follows:

Wang RH, Korotchenko A, Hurd Clarke L, Mortenson WB, Mihailidis A. Power mobility with collision avoidance for older adults: User, caregiver, and prescriber perspectives. J Rehabil Res Dev. 2013;50(9):1287-1300. http://dx.doi.org/10.1682/JRRD.2012.10.0181

ResearcherID/ORCID: Rosalie H. Wang, BSc (OT), PhD: D-3743-2011; Laura Hurd Clarke, MSW, PhD: L7457-2013; Alex Mihailidis, PhD, PEng: D-3759-2011; W. Ben Mortenson, BSc (OT), MSc, PhD: L-7441-2013

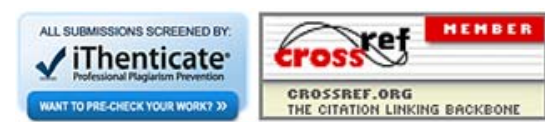

